\title{
THE DRUG COMPLIANCE AMONG PATIENTS WITH DIABETES IN SRI LANKAN SET'TING
}

\author{
Gunathilake $\mathrm{G}^{1}$, Kottahachchi $\mathrm{D}^{2}$, Siyambalapitiya $\mathrm{S}^{3}$.
}

${ }^{1}$ Department of public health, The Faculty of Medicine, The University of Kelaniya, ${ }^{2}$ The Department of physiology, The faculty of Medicine, The University of Kelaniya, ${ }^{3}$ Diabetes and Endocrine unit, North Colombo Teaching Hospital, Ragama.

\section{ABSTRACT}

Introduction: Diabetes mellitus is a chronic non-communicable disease that is associated with vascular complications. Treatment of diabetes complications are expensive and it is much more expensive than the treatment for the disease itself. However, most of these vascular complications can be prevented with good glycaemic control and to achieve this, compliance to medication is essential. Similar to most of the other chronic diseases, drug compliance has been poor among patients with diabetes. The objective of this study was to assess the level of drug compliance and the factors that influence the drug compliance among Sri Lankan patients with diabetes.

Method: Three sets of patients were recruited from three different clinical settings, the diabetes clinic, the medical clinics and the general practitioner clinics in the community. Data were collected using an interviewer administered questionnaire.

Results: There were 247 patients recruited for the study and majority were females $(80.2 \%)$. The majority (79.4\%) attended the clinics regularly. However, a good compliance to medication was seen in $38.4 \%$ of patients. The large number of tablets that they need to swallow, fear of side effects with long-term drug intake and unclear instructions are the main reasons for poor drug compliance among our patients.

Conclusions: Similar to most of the previously done studies, drug compliance is poor among our patients with diabetes. However, the compliance can be improved by avoiding polypharmacy by using combined pills and with proper patient education regarding drug intake and the side-effects.

Short title: Drug compliance

Keywords: Diabetes mellitus, drug compliance, Sri Lanka.

\section{INTRODUCTION}

Diabetes mellitus is a complex, chronic non-communicable disease, which is becoming a rapidly escalating epidemic in the developing and developed countries throughout the world. Due to the increasing prevalence of obesity and growing older population, the prevalence is expected to be increased even further and the world prevalence of diabetes among adults (aged 20-79 years) is projected to be $7.7 \%$ (439 million) by 2030 . Between 2010 and 2030 , there will be a $69 \%$ increase in numbers of adults with diabetes in developing countries and a 20\% increase in developed countries (1) The age-standardized prevalence of diabetes mellitus increased from $2.5 \%$ in 1990 to $8.5 \%$ in 2000 , in the temporal rural community, Sri Lanka (2). According to the recent studies, the prevalence of diabetes mellitus is $14.2 \%$ for men $13.5 \%$ for women (3-4). Available diabetes prevalence data in Sri Lanka show a definite upward trend over the last 18 years. According to the recently published data, Sri Lanka is among the countries with the highest prevalence rates in the world with an estimated figure of 2.8 million adults with diabetes in Sri Lanka (5). Due to the increment of the prevalence of diabetes, it is expected to have a higher number of patients with the complications of diabetes; microvascular disease and cardiovascular disease in our community (6).

Diabetes requires constant attention to diet, exercise, glucose monitoring, and medication to achieve good glycemic control. Self-management is highlighted recently in the long-term management of diabetes. Compliance to prescribed medication is a crucial factor in delivering a good glycemic control in order to minimize longterm complications as well as to reduce the economic burden associated with the care for diabetes. Therapeutic non-compliance has been associated with additional, 
urgent care-visits, hospitalizations, and higher treatment costs. A systemic review has revealed that nonadherence to oral medications in type 2 diabetes is ranging from 36 to $93 \%$ in patients who remains on treatment for 6-24 months and $62-64 \%$ to insulin treatment (7). In clinical practice, the most commonly used definition of compliance is defined as "patient's behaviors (in terms of taking medication, following diets, or executing lifestyle changes) coincide with healthcare providers' recommendations for health and medical advice (8). Several factors determine the patient's compliance to healthcare provider's instructions and recommendations. These are categorized as patient-centered factors, therapy-related factors, healthcare system factors, social and economic factors, and disease factors. Patientcentered factors are demographic factors such as age, ethnicity, gender, education, and marital status. Psychological factors such as patient's beliefs, motivation and negative attitude towards therapy also play an important role. The Patient-prescriber relationship, health literacy, and patient's knowledge are also important. Therapy-related factors such as route of administration, treatment complexity, duration of treatment, medication side effects, the degree of behavioral change required, the taste of medication and requirement for drug storage can also influence the patient's behavior towards good compliance. The main factors identified relating to healthcare systems are the availability and the accessibility. Socio-economic factors such as time commitment, the cost of therapy, income and social support also have a big impact on the compliance to chronic disease such as diabetes. Disease factors include the diseases with fluctuation or absence of symptoms having a poor compliance (9).

Worldwide, a number of researchers have studied the degree of drug compliance and the factors that influence the drug compliance in patients with diabetes and overall, the drug compliance has been poor (7). However, there is minimal data available regarding the drug compliance of diabetes mellitus and factors affects the drug compliance in Sri Lankan patients. This study was carried out to establish the degree of compliance to medication in diabetic patients in Sri Lankan clinic set up and the community, and also to identify the major factors affect the compliance in our patients in order to see how the compliance can be improved in Sri Lankan community and the clinic set up.

\section{METHOD}

The study was conducted in North Colombo Teaching Hospital (NCTH), Ragama (a tertiary care hospital). Ethical approval for the study was obtained from Faculty of Medicine, University of Kelaniya. Data collection was also done from 2 community clinics in 2 Medical Office of Health $(\mathrm{MOH})$ areas, Ja-Ela and Katana. In order to obtain a proper cross-section of the diabetes population, we recruited a total number of 247 diabetic patients, 104 managed in the diabetes clinic at NCTH and 92 patients who were managed in the medical clinics at NCTH, and 51 patients from the community (Katana and Ja-Ela $\mathrm{MOH}$ areas) who received treatment from the general practitioners (GPs). Patients from the diabetes clinic and medical clinics were selected randomly using the clinic registration numbers. Two clusters of patients receiving treatment from GPs were randomly selected from the 2 community clinics at Katana and Ja-Ela $\mathrm{MOH}$ area. After obtaining informed consent from the recruited patients, demographic data, data related to drug compliance and the reasons for noncompliance were collected using a pretested interviewer-administered questionnaire. Statistical analysis was done using SPSS statistical software. A descriptive analysis was done to summarize demographic and for most of the categorical data and Chi-square test was used to check the association of the categorical variable with the drug compliance.

\section{RESULTS}

There were 247 patients recruited for the study, of which $42.1 \%(n=104)$ from the hospital diabetic clinic, $37.2 \%$ from the medical clinics $(n=92)$ and $20.6 \%$ $(n=51)$ from the community. The mean age of the sample population was 57.3 years $(S D \pm 10.75)$ and the majority were females (80.2\%). 193 $(76.3 \%)$ had an education level above grade 6 , but only $17 \%(\mathrm{n}=43)$ were employed. $56.5 \% \quad(n=143)$ had a monthly income below 10000 rupees. Mean duration of diabetes was 7.67 years $(\mathrm{SD} \pm 5.81)$ in our sample and $181(71.5 \%)$ had associated other chronic illnesses (Table 1$)$.

From our patient population, 87\% $(n=215)$ of the patients were managing their diabetes only with oral hypoglycaemic agents, while $8.9 \%$ $(n=22)$ were on both oral medications and insulin for their diabetes control.

The majority of the patients $(79.4 \%$, $\mathrm{n}=201$ ) attended the clinics regularly. For the minority of who were not attending, long waiting time in the queue was the commonest reason $(59.6 \%)$ for not attending the clinic regularly. Good adherence to treatment was reported by $38.4 \%$ $(n=95)$ of the patients and $61.6 \%$ (152) had poor compliance out of which, $7.2 \%(n=18)$ never adhered to treatment. $40.7 \%(\mathrm{n}=81)$ of the females had good compliance to treatment, whereas only $29.2 \%$ $(n=14)$ of males had good compliance to treatment. The majority of the patients $(59.9 \%)$ age less than 60 years had good compliance to recommended treatment when compared to patients more than 60 years of age $(42.1 \%)$.

Education level did not have a statistically significant association with good compliance $(p=0.613)$. However, the patients who we attending diabetes clinic follow-up had better compliance $(51.6 \%)$ than patients from the medical clinic in the hospital $(35.8 \%)$ and private sector clinics (12.6\%). Diabetic patients with other chronic diseases adhered to treatment better $(63.1 \%)$ than those with diabetes alone $(55.1 \%)$. Out of the patients who had poor compliance $(n=152,61.6 \%), 151$ patients $(99.3 \%)$ took a lower dose of medication than recommended and 
Table 1: socio-demographic characteristics

Category of variables

Number (N)

Percentage ( $\%)$

\begin{tabular}{lrc}
\hline Patient number & 247 & $100 \%$ \\
\hline Gender - & & \\
Females & 203 & $80.2 \%$ \\
Males & 44 & $19.8 \%$ \\
\hline Mean age (years) & $57.3(+/-10.75)$ & \\
\hline
\end{tabular}

Education level

Up to grade 6

Grade 6 and above

60

193

$23.7 \%$

$76.3 \%$

\begin{tabular}{lcc}
\hline Employed & 43 & $17 \%$ \\
\hline $\begin{array}{l}\text { Mean duration of diabetes } \\
\text { (years) }\end{array}$ & $7.87(+/-5.81)$ & \\
\hline $\begin{array}{l}\text { Associated other chronic } \\
\text { illnesses }\end{array}$ & 181 & $71.5 \%$ \\
\hline
\end{tabular}

$123(80.9 \%)$ didn't take medication on time and $122(80.2 \%)$ didn't understand the instructions clearly. Lower doses of medication were ingested due to difficulty in ingesting a large number of pills in $28.4 \%$ patients, due long term and short term drug side effects in $27.8 \%$ patients and due to unclear instructions in $27.8 \%$.

Out of the 32 patients who were on insulin $24(75 \%)$ had always been compliant with treatment and only 2 $(0.6 \%)$ were never compliant. Lower doses of insulin were injected by 4 patients, lower frequency of injections was taken by 7 patients, and 1 patient has never taken insulin despite prescribing it. In 3 patients, the compliance to insulin therapy was due to unclear instructions and the lower doses of insulin were taken due to short-term and long-term side effects by 6 patients.

\section{DISCUSSION}

Our study included a cross-section of patients with diabetes from different clinic settings in Sri Lanka. Despite regular clinic attendance, noncompliance to medication was high $(62 \%)$ among our patients with diabetes. Females and the patients where the age less than 60 years were better compliant with medication.
Attending specialist diabetes clinics also seems to have a positive influence on drug compliance. The poor compliance to medication in our community is due to the unclear instructions regarding medication intake, side effects of the drugs and the large number of tablets that they have to swallow. Even for insulin, the poor compliance has been due to unclear instructions and due to the side effects.

Several studies have demonstrated better drugs compliance among females compared to males $(10,11)$ and our patient population also demonstrated the same. However, there are studies, which have demonstrated the opposite (12). Therefore, gender has not been taken as a good predictor of compliance because of these inconsistent conclusions.

The majority of studies have demonstrated better compliance to medication among elderly patients compared to younger patients $(12,13)$. Poor compliance among older patients was mainly non-intentional and it was due to the problems of vision, hearing, and memory. In addition, they may therapy instructions due to cognitive impairment and also the physical difficulties, such as having problems in have more difficulties in following swallowing tablets, opening drug containers, handling small tablets, distinguishing colors or identifying markings on drugs. Therefore, with the necessary additional help from healthcare providers and the family members, the compliance to medication is likely to be even better. However, our results are contradictory to the results of most of the studies, where our younger patients are better compliant with medication compared to older population (age above 60 years.

There was no statically significant association with the education level in our study. Several studies found a positive correlation with drug compliance and the level of education $(14,15)$, and there are studies where they found no association (16). Intuitively, it may be expected that patients with higher educational level have better knowledge about the disease and therapy and therefore to be more compliant. However, there are studies where they have demonstrated better compliance among diabetes patients with lower education (17). It can be argued that patients with lower educational level may be having more trust in physicians' advice. Our study didn't demonstrate a significant association between the level of education and the degree of drug compliance. All these evidences suggest that educational level may not be a good predictor of therapeutic compliance for medication.

Diabetic clinic follow-up patients had better compliance than the patients attending general medical clinics and community-level clinics and it is very likely to be due to the more focused and disease-oriented health education and treatment that is given in these specialized clinics. This highlights the importance of separate diabetic clinics in hospitals in this era of high disease burden to improve the quality of care.

Poor compliance to treatment was multifaceted. Lower doses of medication were ingested due to difficulty in ingesting a large number of pills, due long term and short term drug side effects and due to unclear instructions in our study. Complex 
treatment is believed to threaten the patient's compliance. However, compliance does not seem to correlate with the number of drugs prescribed (18), but the number of dosing times of the prescribed medications (19) and compliance to medication could be considerably improved by simplifying the medication dosing frequencies of the individual drugs. Side effects threaten patient's compliance. Physical discomfort associated with side effects, skepticism about the efficacy of the medication, and loss of confidence and the trust with the prescriber after the side effects (20) explain the reasons for poor compliance with side effects. Poor communication with healthcare providers has a negative effect on patient's compliance (15). In order to achieve good compliance, it is essential that a good and healthy relationship is built between patients and the health care providers during treatment planning. Involvement of the patients during treatment and planning and explaining the potent side effects of treatment will enhance the compliance to medication and the treatment plan.

The studies have demonstrated that the lack of accessibility to healthcare (21), long waiting time for clinic visits (22), and unhappy clinic visits (23) contribute to the poor patient compliance. In our patient cohort, long queues and the waiting time was one of the main reasons for poor regular clinic attendance. Proper organization, development of the infrastructure and improving the efficiency of health care delivery will be helpful in improving the patient compliance.radioiodine, post thyroidectomy) and in the prevalence of diabetes as well as impaired fasting glucose ( $\mathrm{p}-0.079$, $\mathrm{p}$ 0.109). Even though Age, sedentary life style and family history were strongly associated with the development of diabetes mellitus, body mass index and hypertension did not show a significant correlation with the development of diabetes in our study group. Our study did not reveal an increased risk microvascular complications among hypothyroid patients with diabetes.

\section{CONCLUSION}

Non-compliance to anti-diabetic medications is high among patients with diabetes in Sri Lanka. Considering the epidemic nature of this problem, it is essential that the policy makers make a sincere effort to identify the barriers in individual settings and find remedies in order to face this enormous burden of diabetes in the future.

\section{REFERRENCES}

1. Shaw JE, Sicre RA, Zimmet PZ. Global estimates of the prevalence of diabetes for 2010 and 2030. Diabetes research and clinical practice 2010: 87(1): 4-14.

2. Illangasekera $\mathrm{U}$, Rambodagalla $\mathrm{S}$, Tennakoon S. Temporal trends in the prevalence of diabetes mellitus in a rural community Sri Lanka. The journal of the Royal Society for the Promotion of Health 2004; 124(2): 92-94.

3. Illangasekara U, Nugegoda DB, Perera LS. prevalence of diabetes mellitus and impaired glucose tolerance in a rural Sri Lankan community. Ceylon medical journal 1993; 38(3): 123-126.

4. Wijewardene K, Mohideen MR, Mendis S, Fernando DS, Kulathilaka T, Weerasekara D, Uluwitta P. Prevalence of hypertension, diabetes and obesity: baseline findings of a population-based survey in 4 provinces in Sri Lanka. Ceylon medical journal 2005; 50(2): 62-70.

5. Katulanda P, Sheriff MHR, Matthews DR. The diabetes epidemic in Sri Lankaa growing problem. The Ceylon medical journal 2006; 59(1): 26-28.

6. Fernando DJ, Siribaddana S, Perera N, Perera S, de Silva D. The prevalence of macrovascular disease and lipid abnormalities amongst diabetic patients in Sri Lanka. Postgraduate medical journal 1993; 69: 557-561.

7. Joyce A. Cramer. A Systematic Review of Adherence with Medications for Diabetes. Diabetes Care 2004; 27(5): 1218-1224.

8. Sackett DL, Haynes RB. Compliance with therapeutic regimens. Baltimore: John Hopkins University Press 1976: 1-6.

9. Jing Jin, Grant Edward Sklar, Vernon Min Sen Oh, Shu Chuen Li. Factors affecting therapeutic compliance: A review from the patient's perspective. Therapeutics and Clinical Risk Management 2008; 4(1): 269-286.

10. Lertmaharit S, Kamol-Ratankul P, Sawert H, Jittimanee S, Wangmanee S. Factors associated with compliance among tuberculosis patients in Thailand. Journal of the Medical Association of Thailand 2005; 88(4): 149-156.

11. Kiortsis DN, Giral P, Bruckert E, Turpin G. Factors associated with low compliance with lipid-lowering drugs in hyperlipidemic patients. Journal of Clinical Pharmacology and Therapeutics 2000; 25: 445-451.
12. Hertz RP, Unger AN, Lustik MB. Adherence with pharmacotherapy for type 2 diabetes: a retrospective cohort study of adults with employer-sponsored health insurance. Clinical Therapeutics 2005; 27: 1064-1073.

13. Senior V, Marteau TM, Weinman J. Selfreported adherence to cholesterollowering medication in patients with familial hypercholesterolaemia: the role of illness perceptions. Cardiovascular Drugs and Therapy 2004; 18: 475-481.

14. Ghods AJ, Nasrollahzadeh D. Noncompliance with immunosuppressive medications after renal transplantation. Experimental and Clinical Transplantion 2003; 1: 39-47.

15. Apter AJ, Reisine ST, Affleck G, Barrows E, ZuWallack RL. Adherence with twice-daily dosing of inhaled steroids. Socioeconomic and healthbelief differences. American Journal of Respiratory and Critical Care Medicine 1998; 157: 1810-1817.

16. Horne R, Weinman J. Patients' beliefs about prescribed medicines and their role in adherence to treatment in chronic physical illness. Journal of Psychosomatic Research 1999; 47: 555-567.

17. Kyngas H, Lahdenpera T. Compliance of patients with hypertension and associated factors. Journal of Advanced Nursing 1999; 29: 832-839.

18. Grant RW, Devita NG, Singer DE, et al. Polypharmacy and medication adherence in patients with type 2 diabetes. Diabetes Care 2003; 26: 1408-1412.

19. Iskedjian M, Einarson TR, MacKeigan LD, Shear N, Addis A, Mittmann N, et al. Relationship between daily dose frequency and adherence to antihypertensive pharmacotherapy: evidence from a meta-analysis. Clinical Therapeutics 2002; 24: 302-316.

20. Christensen DB. Drug-taking compliance: a review and synthesis. Health Service Research 1978; 13: 171-187.

21. Ponnusankar S, Surulivelrajan M, Anandamoorthy N, Suresh B. Assessment of the impact of medication counseling on patients' medication knowledge and compliance in an outpatient clinic in South India. Patient Education and Counselling 2004; 54: 55-60.

22. Balkrishnan R, Rajagopalan R, Camacho FT, Huston SA, Murray FT, Anderson RTl. Predictors of medication adherence and associated health care costs in an older population with type 2 diabetes mellitus: a longitudinal cohort study. Clinical Therapeutics 2003; 25: 2958-2971.

23. Lawson VL, Lyne PA, Harvey JN, Bundy CE. Understanding why people with 
type 1 diabetes do not attend for specialist advice: a qualitative analysis of the views of people with insulin-dependent diabetes who do not attend diabetes clinic. Journal of Health Psychology 2005; 10: 409-423. 